\title{
On an Extension of Kummer's Second Theorem
}

\author{
Medhat A. Rakha, ${ }^{1,2}$ Mohamed M. Awad, ${ }^{2}$ and Arjun K. Rathie ${ }^{3}$ \\ ${ }^{1}$ Department of Mathematics and Statistics, College of Science, Sultan Qaboos University, P.O. Box 36 (123), Alkhoud, Muscat, Oman \\ ${ }^{2}$ Department of Mathematics, Faculty of Science, Suez Canal University, Ismailia 41511, Egypt \\ ${ }^{3}$ Department of Mathematics, School of Mathematical and Physical Sciences, Central University of Kerala, \\ Riverside Transit Campus, Padennakkad P.O. Nileshwar, Kasaragod, Kerala 671 328, India
}

Correspondence should be addressed to Medhat A. Rakha; medhat@squ.edu.om

Received 4 December 2012; Accepted 26 February 2013

Academic Editor: Adem Kiliçman

Copyright (c) 2013 Medhat A. Rakha et al. This is an open access article distributed under the Creative Commons Attribution License, which permits unrestricted use, distribution, and reproduction in any medium, provided the original work is properly cited.

The aim of this paper is to establish an extension of Kummer's second theorem in the form $e^{-x / 2}{ }_{2} F_{2}\left[\begin{array}{ccc}a, 2+d ; & \\ 2 a+2, & d ;\end{array}\right]=$ ${ }_{0} F_{1}\left[{ }_{a+3 / 2 ;}^{-;} x^{2} / 16\right]+((a / d-1 / 2) /(a+1)) x_{0} F_{1}\left[{ }_{a+3 / 2 ;}^{-;} x^{2} / 16\right]+\left(c x^{2} / 2(2 a+3)\right)_{0} F_{1}\left[\begin{array}{c}-; \\ a+5 / 2 ;\end{array} x^{2} / 16\right]$, where $c=(1 /(a+1))(1 / 2-a / d)+$ $a / d(d+1), d \neq 0,-1,-2, \ldots$. For $d=2 a$, we recover Kummer's second theorem. The result is derived with the help of Kummer's second theorem and its contiguous results available in the literature. As an application, we obtain two general results for the terminating ${ }_{3} F_{2}(2)$ series. The results derived in this paper are simple, interesting, and easily established and may be useful in physics, engineering, and applied mathematics.

\section{Introduction}

The generalized hypergeometric function ${ }_{p} F_{q}$ with $p$ numerator and $q$ denominator parameters is defined by [1]

$$
\begin{aligned}
{ }_{p} F_{q}\left[\begin{array}{c}
a_{1}, \ldots, a_{p} ; \\
b_{1}, \ldots, b_{q} ;
\end{array}\right] & ={ }_{p} F_{q}\left[a_{1}, \ldots, a_{p} ; b_{1}, \ldots, b_{q} ; z\right] \\
& =\sum_{n=0}^{\infty} \frac{\left(a_{1}\right)_{n} \cdots\left(a_{p}\right)_{n}}{\left(b_{1}\right)_{n} \cdots\left(b_{q}\right)_{n}} \frac{z^{n}}{n !}
\end{aligned}
$$

where $(a)_{n}$ denotes Pochhammer's symbol (or the shifted or raised factorial, since $(1)_{n}=n$ !) defined by

$$
(a)_{n}= \begin{cases}a(a+1) \cdots(a+n-1), & n \in \mathbb{N}, \\ 1, & n=0 .\end{cases}
$$

Using the fundamental properties of Gamma function $\Gamma(a+$ $1)=a \Gamma(a),(a)_{n}$ can be written in the form

$$
(a)_{n}=\frac{\Gamma(a+n)}{\Gamma(a)},
$$

where $\Gamma$ is the familiar Gamma function.
It is not out of place to mention here that whenever a generalized hypergeometric or hypergeometric function ${ }_{2} F_{1}$ reduces to Gamma function, the results are very important from the applicative point of view. Thus, the classical summation theorem for the series ${ }_{2} F_{1}$ such as those of Gauss, Gauss second, Kummer, and Bailey plays an important role in the theory of hypergeometric series. For generalization and extensions of these classical summation theorems, we refer to $[2,3]$.

By employing the above mentioned classical summation theorems, Bailey [4] had obtained a large number of very interesting results (including results due to Ramanujan, Gauss, Kummer, and Whipple) involving products of generalized hypergeometric series.

On the other hand, from the theory of differential equations, Kummer [5] established the following very interesting and useful result known in the literature as Kummer's second theorem:

$$
e^{-x / 2}{ }_{1} F_{1}\left[\begin{array}{cc}
a ; & \\
2 a ;
\end{array}\right]={ }_{0} F_{1}\left[\begin{array}{ll}
-; & \frac{x^{2}}{16} \\
a+\frac{1}{2} ; &
\end{array}\right] .
$$


Bailey [4] established the result (4) by employing the Gauss second summation theorem, and Choi and Rathie [6] established the result (4) (of course, by changing $x$ to $2 x$ ) by employing the classical Gauss summation theorem. From (4), Rainville [7] deduced the following two useful and classical results:

$$
{ }_{2} F_{1}\left[\begin{array}{lll}
-2 n, & a ; & \\
2 a ; & & 2
\end{array}\right]=\frac{(1 / 2)_{n}}{(a+1 / 2)_{n}}, \quad\left(n \in \mathbb{N}_{0}:=\mathbb{N} \cup\{0\}\right),
$$$$
{ }_{2} F_{1}\left[\begin{array}{lll}
-2 n-1, a ; & \\
2 a ; &
\end{array}\right]=0, \quad\left(n \in \mathbb{N}_{0}\right)
$$

Using (5) and (6), it is not difficult to establish the following transformation due to Kummer:

$$
\begin{aligned}
& (1-x)_{2}^{-r} F_{1}\left[\begin{array}{lll}
r, & m ; & \\
& & -\frac{2 x}{1-x} \\
2 m ; &
\end{array}\right] \\
& ={ }_{2} F_{1}\left[\begin{array}{ll}
\frac{1}{2} r, & \frac{1}{2} r+\frac{1}{2} ; \\
m+\frac{1}{2} ; & x^{2} \\
&
\end{array}\right] \text {. }
\end{aligned}
$$

In 1995, Rathie and Nagar [8] obtained two results closely related to Kummer's second theorem (4); one of those results is given below:

$$
\begin{aligned}
e^{-x / 2}{ }_{1} F_{1}\left[\begin{array}{ll}
a ; \\
2 a+1 ;
\end{array}\right]= & { }_{0} F_{1}\left[\begin{array}{ll}
-; & \frac{x^{2}}{16} \\
a+\frac{1}{2} ;
\end{array}\right] \\
& -\frac{x}{2(2 a+1)}{ }_{0} F_{1}\left[\begin{array}{ll}
-; & \frac{x^{2}}{16} \\
a+\frac{3}{2} ; &
\end{array}\right] .
\end{aligned}
$$

In 2010, Kim et al. [1] have generalized the Kummer's second theorem and obtained explicit expressions of

$$
e^{-x / 2}{ }_{1} F_{1}\left[\begin{array}{l}
a ; \\
2 a+j ;
\end{array}\right]
$$

for $j=0, \pm 1, \ldots, \pm 5$ by employing the generalized Gauss second summation theorem obtained earlier by Lavoie et al. [9].
We, however, would like to mention one of their results which we will require in our present investigation:

$$
\begin{aligned}
& e^{-x / 2}{ }_{1} F_{1}\left[\begin{array}{ll}
a ; & x \\
2 a+2 ; &
\end{array}\right] \\
& ={ }_{0} F_{1}\left[\begin{array}{ll}
-; & \frac{x^{2}}{16} \\
a+\frac{3}{2} ;
\end{array}\right]-\frac{x}{2(a+1)}{ }_{0} F_{1}\left[\begin{array}{ll}
-; & \frac{x^{2}}{16} \\
a+\frac{3}{2} ;
\end{array}\right] \\
& +\frac{x^{2}}{4(a+1)(2 a+3)}{ }_{0} F_{1}\left[\begin{array}{cc}
-; & \frac{x^{2}}{16} \\
a+\frac{5}{2} ; &
\end{array}\right.
\end{aligned}
$$

In 2008, Rathie and Pogàny [10] established a new summation formula for ${ }_{3} F_{2}(1 / 2)$ and, as an application, obtained the following result which is known as an extension of Kummer's second Theorem (4):

$$
\begin{aligned}
& e^{-x / 2}{ }_{2} F_{2}\left[\begin{array}{ll}
a, & 1+d ; \\
2 a+1, & d ;
\end{array}\right] \\
& ={ }_{0} F_{1}\left[\begin{array}{lr}
-; & \frac{x^{2}}{16} \\
a+\frac{1}{2} ;
\end{array}\right] \\
& -\frac{x(1-2 a / d)}{2(2 a+1)}{ }_{0} F_{1}\left[\begin{array}{l}
-; \\
a+\frac{3}{2} ;
\end{array}\right],
\end{aligned}
$$

for $d \neq 0,-1,-2, \ldots$.

It is noted that if in (11) we set $d=2 \alpha$, we immediately recover Kummer's second Theorem (4).

Very recently Rakha [11] rederived the result (11) in its equivalent form by employing the classical Gauss summation theorem, and Kim et al. [12] derived (11) in a very elementary way and, as an application, obtained the following two elegant results:

$$
{ }_{3} F_{2}\left[\begin{array}{lll}
-2 n, & a, 1+d ; \\
2 a+1, & d ; & 2
\end{array}\right]=\frac{(1 / 2)_{n}}{(a+1 / 2)_{n}}, \quad\left(n \in \mathbb{N}_{0}\right),
$$

$$
{ }_{3} F_{2}\left[\begin{array}{lll}
-2 n-1, & a, 1+d ; \\
2 a+1, & d ; & 2
\end{array}\right]=\frac{(1-2 a / d)}{(2 a+1)} \frac{(3 / 2)_{n}}{(a+3 / 2)_{n}},
$$

It is interesting to mention here that the right-hand side of (12) is independent of $d$, where $d \neq 0,-1,-2, \ldots$. 
Remark 1. (a) In (12) and (13), if we set $d=2 a$, we recover (5) and (6), respectively.

(b) Using (12) and (13), Kim et al. [12] have obtained the following extension of transformation (7) due to Kummer:

$$
\begin{aligned}
& (1-x)^{-r}{ }_{3} F_{2}\left[\begin{array}{lll}
r, & m, d+1 ; & \\
2 m+1, d ; & -\frac{2 x}{1-x}
\end{array}\right] \\
& ={ }_{2} F_{1}\left[\begin{array}{l}
\frac{1}{2} r, \quad \frac{1}{2} r+\frac{1}{2} ; \\
m+\frac{1}{2} ;
\end{array}\right] \\
& +\frac{x r(1-2 m / d)}{(2 m+1)}{ }_{2} F_{1}\left[\begin{array}{ll}
\frac{1}{2} r+\frac{1}{2}, & \frac{1}{2} r+1 ; \\
m+\frac{3}{2} ; & x^{2}
\end{array}\right]
\end{aligned}
$$

for $d \neq 0,-1,-2, \ldots$

The aim of this paper is to establish another extension of Kummer's second Theorem (4) by employing the known results (4), (8), and (10). As an application, we mention two interesting results for the terminating ${ }_{3} F_{2}(2)$ series. The results established in this paper are simple, interesting, and easily established and may be useful in physics, engineering, and applied mathematics.

\section{Main Result}

The result to be established in this paper is as follows:

$$
\begin{aligned}
& e^{-x / 2}{ }_{2} F_{2}\left[\begin{array}{lll}
a, & 2+d ; & \\
2 a+2, & d ; &
\end{array}\right] \\
& ={ }_{0} F_{1}\left[\begin{array}{cc}
-; & \frac{x^{2}}{16} \\
a+\frac{3}{2} ; &
\end{array}\right] \\
& +\frac{(a / d-1 / 2)}{(a+1)} \times{ }_{0} F_{1}\left[\begin{array}{ll}
-; & \frac{x^{2}}{16} \\
a+\frac{3}{2} ; &
\end{array}\right] \\
& +\frac{c x^{2}}{2(2 a+3)}{ }_{0} F_{1}\left[\begin{array}{ll}
-; & \\
a+\frac{5}{2} ; & \frac{x^{2}}{16}
\end{array}\right]
\end{aligned}
$$

where $d \neq 0,-1,-2, \ldots$ and $c$ is given by $c=(1 /(a+1))(1 / 2-$ $a / d)+a / d(d+1)$.
2.1. Derivation. In order to derive (15), we proceed as follows. Denoting the left-hand side of (15) by $S$ and expressing ${ }_{2} F_{2}$ as a series with the help of (1), we have

$$
S=e^{-x / 2} \sum_{n=0}^{\infty} \frac{(a)_{n}}{(2 a+2)_{n}} \frac{x^{n}}{n !}\left\{\frac{(2+d)_{n}}{(d)_{n}}\right\} .
$$

Now, it is not difficult to see that

$$
\frac{(2+d)_{n}}{(d)_{n}}=1+\frac{2}{d} n+\frac{n(n-1)}{d(d+1)}
$$

we have

$$
S=e^{-x / 2} \sum_{n=0}^{\infty} \frac{(a)_{n}}{(2 a+2)_{n}} \frac{x^{n}}{n !}\left\{1+\frac{2}{d} n+\frac{n(n-1)}{d(d+1)}\right\} .
$$

Separating (18) into three terms, we have

$$
\begin{gathered}
S=e^{-x / 2}\left[\sum_{n=0}^{\infty} \frac{(a)_{n}}{(2 a+2)_{n}} \frac{x^{n}}{n !}+\frac{2}{d} \sum_{n=1}^{\infty} \frac{(a)_{n}}{(2 a+2)_{n}} \frac{x^{n}}{(n-1) !}\right. \\
\left.+\frac{1}{d(d+1)} \sum_{n=2}^{\infty} \frac{(a)_{n}}{(2 a+2)_{n}} \frac{x^{n}}{(n-2) !}\right] .
\end{gathered}
$$

For the second and third terms on the right-hand side of (19), changing $n$ to $n+1$ and $n$ to $n+2$, respectively, and making use of the following results:

$$
\begin{gathered}
(a)_{n+1}=a(a+1)_{n}, \\
(2 a+2)_{n+1}=(2 a+2)(2 a+3)_{n}, \\
(a)_{n+2}=a(a+1)(a+2)_{n}, \\
(2 a+2)_{n+2}=(2 a+2)(2 a+3)(2 a+4)_{n},
\end{gathered}
$$

we have, after some simplification,

$$
\begin{aligned}
S=e^{-x / 2}\left[\sum_{n=0}^{\infty} \frac{(a)_{n}}{(2 a+2)_{n}} \frac{x^{n}}{n !}+\frac{a x}{d(a+1)} \sum_{n=0}^{\infty} \frac{(a+1)_{n}}{(2 a+3)_{n}} \frac{x^{n}}{n !}\right. \\
\left.+\frac{a x^{2}}{2 d(d+1)(2 a+3)} \sum_{n=0}^{\infty} \frac{(a+2)_{n}}{(2 a+4)_{n}} \frac{x^{n}}{n !}\right] .
\end{aligned}
$$

Now, summing up the series with the help of (1), we have

$$
\begin{aligned}
S= & e^{-x / 2}{ }_{1} F_{1}\left[\begin{array}{l}
a ; \\
2 a+2 ;
\end{array}\right] \\
& +\frac{a x}{d(a+1)} e_{1}^{-x / 2} F_{1}\left[\begin{array}{ll}
a+1 ; & x \\
2 a+3 ;
\end{array}\right] \\
& +\frac{a x^{2}}{2 d(d+1)(2 a+3)} e^{-x / 2}{ }_{1} F_{1}\left[\begin{array}{cc}
a+2 ; & x \\
2 a+4 ; &
\end{array}\right] .
\end{aligned}
$$

Finally, observing the right-hand side of (22), we see that the first, second, and third expressions can now be evaluated with the help of the results (10), (8), and (4), respectively, and, after some simplification, we arrive at the desired result (15). This completes the proof of (15). 


\section{New Results for Terminating ${ }_{3} F_{2}(2)$}

In this section, from our newly obtained result (15), we will establish two new results for the terminating ${ }_{3} F_{2}$ series. These are

$$
\begin{array}{r}
{ }_{3} F_{2}\left[\begin{array}{ccc}
-2 n, & a, & 2+d ; \\
2 a+2, & d ; & 2
\end{array}\right]=\frac{(1 / 4 c+1)_{n}(1 / 2)_{n}}{(1 / 4 c)_{n}(a+3 / 2)_{n},} \\
\left(n \in \mathbb{N}_{0}\right), \\
{ }_{3} F_{2}\left[\begin{array}{ccc}
-2 n-1, & a, 2+d ; \\
2 a+2, & d ; & 2
\end{array}\right]=\frac{(d-2 a)}{d(a+1)} \frac{(3 / 2)_{n}}{(a+3 / 2)_{n}}, \\
\left(n \in \mathbb{N}_{0}\right),
\end{array}
$$

where $c=(1 /(a+1))(1 / 2-a / d)+a / d(d+1)$, and $d \neq 0$, $-1,-2,, \ldots$.

3.1. Derivations. In order to derive the results (23), we proceed as follows. Denoting the left-hand side of (15) by $S_{1}$, then expressing both of the functions involved in the series, we have

$$
\begin{aligned}
S_{1} & =\sum_{n=0}^{\infty} \frac{(-1)^{n}}{2^{n}} \frac{x^{n}}{n !} \sum_{m=0}^{\infty} \frac{(a)_{m}(2+d)_{m}}{(2 a+2)_{m}(d)_{m} m !} x^{m} \\
& =\sum_{n=0}^{\infty} \sum_{m=0}^{\infty} \frac{(-1)^{n}(a)_{m}(2+d)_{m}}{2^{n}(2 a+2)_{m}(d)_{m} m ! n !} x^{n+m} .
\end{aligned}
$$

Replacing $n$ by $n-m$ in (24) and using the known result [7, page 56, Lemma 10]:

$$
\sum_{n=0}^{\infty} \sum_{k=0}^{\infty} A(k, n)=\sum_{n=0}^{\infty} \sum_{k=0}^{n} A(k, n-k)
$$

we have

$$
S_{1}=\sum_{n=0}^{\infty} \sum_{m=0}^{n} \frac{(-1)^{n-m}(a)_{m}(2+d)_{m}}{2^{n-m}(2 a+2)_{m}(d)_{m} m !(n-m) !} x^{n} .
$$

Using the identity

$$
(n-m) !=\frac{(-1)^{m} n !}{(-n)_{m}}
$$

we have, after some simplification,

$$
S_{1}=\sum_{n=0}^{\infty} \frac{(-1)^{n} x^{n}}{2^{n} n !} x^{n} \sum_{m=0}^{n} \frac{(-n)_{m}(a)_{m}(2+d)_{m}}{(2 a+2)_{m}(d)_{m} m !} 2^{m} .
$$

Expressing the inner series in the last result, we get

$$
S_{1}=\sum_{n=0}^{\infty} \frac{(-1)^{n} x^{n}}{2^{n} n !} x_{3}^{n} F_{2}\left[\begin{array}{ccc}
-n, & a, 2+d ; \\
2 a+2, & d ; & 2
\end{array}\right] .
$$

Now, separating the ${ }_{3} F_{2}$ into even and odd powers of $x$ and making use of the results:

$$
\begin{gathered}
(2 n) !=2^{2 n} n !\left(\frac{1}{2}\right)_{n}, \\
(2 n+1) !=2^{2 n} n !\left(\frac{3}{2}\right)_{n},
\end{gathered}
$$

we finally have

$$
\begin{aligned}
S_{1}= & \sum_{n=0}^{\infty} \frac{x^{2 n}}{2^{4 n} n !(1 / 2)_{n}}{ }_{3} F_{2}\left[\begin{array}{ccc}
-2 n, & a, 2+d ; & 2 \\
2 a+2, & d ; &
\end{array}\right] \\
& -\sum_{n=0}^{\infty} \frac{x^{2 n+1}}{2^{4 n+1} n !(3 / 2)_{n}}{ }_{3} F_{2}\left[\begin{array}{ccc}
-2 n-1, & a, 2+d ; \\
2 a+2, & d ; & 2
\end{array}\right] .
\end{aligned}
$$

Also, it is not difficult to see that

$$
\begin{aligned}
& { }_{1} F_{2}\left[\begin{array}{cc}
\frac{1}{4 c}+1 ; & \frac{x^{2}}{16} \\
\frac{1}{4 c}, & a+\frac{3}{2} ;
\end{array}\right] \\
& ={ }_{0} F_{1}\left[\begin{array}{c}
-; \\
a+\frac{3}{2} ;
\end{array}\right]+\frac{c x^{2}}{2(2 a+3)}{ }_{0} F_{1}\left[\begin{array}{l}
-; \\
a+\frac{x^{2}}{2} ;
\end{array}\right] .
\end{aligned}
$$

Now, if we denote the right-hand side of (15) by $S_{2}$, then with the help of (32), it can be expressed as

$$
\begin{aligned}
& S_{2}={ }_{1} F_{2}\left[\begin{array}{lr}
\frac{1}{4 c}+1 ; & \frac{x^{2}}{16} \\
\frac{1}{4 c}, & a+\frac{3}{2} ;
\end{array}\right] \\
& +\frac{(a / d-1 / 2)}{(a+1)} x_{0} F_{1}\left[\begin{array}{cr}
-; & \frac{x^{2}}{16} \\
a+\frac{3}{2} ; &
\end{array}\right] .
\end{aligned}
$$

Thus, from (31) and (33), if we equate the coefficients of $x^{2 n}$ and $x^{2 n+1}$ on both sides, we at once arrive at the results (23). This completes the proof.

Remark 2. (a) Setting $d=2 a$ in (15), we immediately recover Kummer's second Theorem (4). Thus, (15) can be regarded as the extension of (4).

(b) Also, if we take $d=2 a$ in (12) and (13), we again at once get the result (5) and (6), respectively. Thus, our results (12) and (13) can be regarded as extensions of (5) and (6). 


\section{Extension of a Transformation due to Kummer}

In this section, we will establish a natural extension of Kummer's transformation:

$$
\begin{aligned}
& (1-x)^{-r}{ }_{3} F_{2}\left[\begin{array}{rr}
r, & m, d+2 ; \\
2 m+2, d ; & -\frac{2 x}{1-x}
\end{array}\right] \\
& ={ }_{3} F_{2}\left[\begin{array}{l}
\frac{1}{2} r, \quad \frac{1}{2} r+\frac{1}{2}, \frac{1}{4 c}+1 ; \\
m+\frac{3}{2}, \frac{1}{4 c} ;
\end{array}\right] \\
& +\frac{x r(d-2 m)}{d(m+1)}{ }_{2} F_{1}\left[\begin{array}{ll}
\frac{1}{2} r+\frac{1}{2}, & \frac{1}{2} r+1 ; \\
m+\frac{3}{2} ; & x^{2}
\end{array}\right],
\end{aligned}
$$

for $d \neq 0,-1,-2, \ldots$ and $c$ is given by

$$
c=\frac{1}{m+1}\left(\frac{1}{2}-\frac{m}{d}\right)+\frac{m}{d(d+1)} .
$$

4.1. Derivation. In order to establish the result (34), we proceed as follows. Denote the left-hand side of (34) by $S_{3}$; we have

$$
S_{3}=(1-x)^{-r}{ }_{3} F_{2}\left[\begin{array}{rrr}
r, & m, d+2 ; & \\
2 m+2, d ; & -\frac{2 x}{1-x}
\end{array}\right] ;
$$

expressing ${ }_{3} F_{2}$ as a series, we have

$$
S_{3}=\sum_{k=0}^{\infty} \frac{(r)_{k}(m)_{k}(d+2)_{k}(-1)^{k} 2^{k} x^{k}}{(2 m+2)_{k}(d)_{k} k !}(1-x)^{-(r+k)} .
$$

Applying the generalized Binomial theorem

$$
(1-z)^{a}=\sum_{n=0}^{\infty} \frac{(-a)_{n}}{n !} z^{n} \quad(|z|<1),
$$

we have

$$
S_{3}=\sum_{k=0}^{\infty} \sum_{n=0}^{\infty} \frac{(r)_{k}(m)_{k}(d+2)_{k}(-2)^{k}}{(2 m+2)_{k}(d)_{k} k ! n !}(r+k)_{n} x^{k+n} .
$$

Using $(r)_{k}(r+k)_{n}=(r)_{k+n}$, we have

$$
S_{3}=\sum_{k=0}^{\infty} \sum_{n=0}^{\infty} \frac{(m)_{k}(d+2)_{k}(-2)^{k}(r)_{k+n}}{(2 m+2)_{k}(d)_{k} k ! n !} x^{k+n} ;
$$

changing $n$ to $n-k$ and using (25), we have

$$
S_{3}=\sum_{n=0}^{\infty} \sum_{k=0}^{n} \frac{(m)_{k}(d+2)_{k}(-2)^{k}(r)_{n}}{(2 m+2)_{k}(d)_{k} k !(n-k) !} x^{n} .
$$

\section{Using}

$$
(n-k) !=\frac{(-1)^{k} n !}{(-n)_{k}} \quad(0 \leq k \leq n),
$$

we have

$$
\begin{aligned}
S_{3} & =\sum_{n=0}^{\infty} \sum_{k=0}^{n} \frac{(m)_{k}(d+2)_{k}(-2)^{k}(r)_{n}(-n)_{k}}{(2 m+2)_{k}(d)_{k} k !(-1)^{k} n !} x^{n} \\
& =\sum_{n=0}^{\infty} \frac{(r)_{n}}{n !} x^{n} \sum_{k=0}^{n} \frac{(-n)_{k}(m)_{k}(d+2)_{k}}{(2 m+2)_{k}(d)_{k} k !} 2^{k} .
\end{aligned}
$$

Expressing the inner series, as ${ }_{3} F_{2}$, we find

$$
S_{3}=\sum_{n=0}^{\infty} \frac{(r)_{n}}{n !} x^{n}{ }_{3} F_{2}\left[\begin{array}{lll}
-n, & m, d+2 ; \\
2 m+2, & d ;
\end{array}\right],
$$

from which, we have

$$
\begin{aligned}
S_{3}= & \sum_{n=0}^{\infty} \frac{(r)_{2 n}}{(2 n) !} x^{2 n}{ }_{3} F_{2}\left[\begin{array}{lll}
-2 n, & m, d+2 ; & 2 \\
2 m+2, & d ;
\end{array}\right] \\
& +\sum_{n=0}^{\infty} \frac{(r)_{2 n+1}}{(2 n+1) !} x^{2 n+1}{ }_{3} F_{2}\left[\begin{array}{lll}
-2 n-1, & m, d+2 ; \\
2 m+2, & d ; & 2
\end{array}\right] .
\end{aligned}
$$

Using the the following identities:

$$
\begin{gathered}
(r)_{2 n}=2^{2 n}\left(\frac{1}{2} r\right)_{n}\left(\frac{1}{2} r+\frac{1}{2}\right)_{n}, \\
(r)_{2 n+1}=r 2^{2 n}\left(\frac{1}{2} r+\frac{1}{2}\right)_{n}\left(\frac{1}{2} r+1\right)_{n}, \\
(2 n) !=2^{2 n}\left(\frac{1}{2}\right)_{n} n !
\end{gathered}
$$

$$
(2 n+1) !=2^{2 n}\left(\frac{3}{2}\right)_{n} n !
$$

together with the ${ }_{3} F_{2}(2)$ result, we have

$$
\begin{aligned}
S_{3}= & \sum_{n=0}^{\infty} \frac{((1 / 2) r)_{n}((1 / 2) r+1 / 2)_{n}}{n !} x^{2 n} \frac{(1 / 4 c+1)_{n}}{(1 / 4 c)_{n}(m+3 / 2)_{n}} \\
& +x \sum_{n=0}^{\infty} \frac{r((1 / 2) r+1 / 2)_{n}((1 / 2) r+1)}{n !} x^{2 n+1} \\
& \times \frac{(d-2 m)}{d(m+1)(m+3 / 2)_{n}}
\end{aligned}
$$




$$
\begin{gathered}
={ }_{3} F_{2}\left[\begin{array}{cc}
\frac{1}{2} r, \frac{1}{2} r+\frac{1}{2}, & \frac{1}{4 c}+1 ; \\
\frac{1}{4 c}, m+\frac{3}{2} ; & x^{2}
\end{array}\right] \\
+\frac{x(d-2 m) r}{d(m+1)}{ }_{2} F_{1}\left[\begin{array}{ll}
\frac{1}{2} r+\frac{1}{2}, & \frac{1}{2} r+1 ; \\
m+\frac{3}{2} ; & x^{2}
\end{array}\right],
\end{gathered}
$$

with

$$
c=\frac{1}{m+1}\left(\frac{1}{2}-\frac{m}{d}\right)+\frac{m}{d(d+1)}, \quad d \neq 0,-1,-2, \ldots .
$$

This completes the proof of (34).

Remark 3. In (34), if we take $d=2 m$, we get (7). Thus, (34) may be regarded as an extension of (7).

\section{Authors' Contribution}

All authors contributed equally to this paper. They read and approved the final paper.

\section{Acknowledgments}

The authors would like to express their sincere gratitude to the referees for their valuable comments and suggestions. They are so much appreciated to the College of Science, Sultan Qaboos University, Muscat, Oman, for supporting the publication charge of this paper.

\section{References}

[1] Y. S. Kim, M. A. Rakha, and A. K. Rathie, "Generalizations of Kummer's second theorem with application," Journal of Computational Mathematics and Mathematical Physics, vol. 50, no. 3, pp. 387-402, 2010.

[2] Y. S. Kim, M. A. Rakha, and A. K. Rathie, "Extensions of certain classical summation theorems for the series ${ }_{2} F_{1},{ }_{3} F_{2}$, and ${ }_{4} F_{3}$ with applications in Ramanujan's summations," International Journal of Mathematics and Mathematical Sciences, vol. 2010, Article ID 309503, 26 pages, 2010.

[3] M. A. Rakha and A. K. Rathie, "Generalizations of classical summation theorems for the series ${ }_{2} F_{1}$ and ${ }_{3} F_{2}$ with applications," Integral Transforms and Special Functions, vol. 22, no. 11, pp. 823-840, 2011.

[4] W. N. Bailey, "Products of generalized hypergeometric series," Proceedings of the London Mathematical Society, vol. 28, no. 1, pp. 242-250, 1928.

[5] E. E. Kummer, “Über die hypergeometridche Reihe," Journal für Die Reine Und Angewandte Mathematik, vol. 15, pp. 39-83, 1836.

[6] J. Choi and A. K. Rathie, "Another proof of Kummer's second theorem," Communications of the Korean Mathematical Society, vol. 13, no. 4, pp. 933-936, 1998.

[7] E. D. Rainville, Special Functions, The Macmillan Company, New York, NY, USA, 1960.
[8] A. K. Rathie and V. Nagar, "On Kummer's second theorem involving product of generalized hypergeometric series," Le Matematiche, vol. 50, no. 1, pp. 35-38, 1995.

[9] J.-L. Lavoie, F. Grondin, and A. K. Rathie, "Generalizations of Watson's theorem on the sum of $\mathrm{a}_{3} F_{2}$," Indian Journal of Mathematics, vol. 34, no. 1, pp. 23-32, 1992.

[10] A. K. Rathie and T. K. Pogány, "New summation formula for ${ }_{3} F_{2}(1 / 2)$ and a Kummer-type II transformation of ${ }_{2} F_{2}(x)$," Mathematical Communications, vol. 13, no. 1, pp. 63-66, 2008.

[11] M. A. Rakha, "A note on Kummer-type II transformation for the generalized hypergeometric function ${ }_{2} F_{2}$," Mathematical Notes, vol. 19, no. 1, pp. 154-156, 2012.

[12] Y. S. Kim, J. Choi, and A. K. Rathie, "Two results for the terminating ${ }_{3} F_{2}(2)$ with applications," Bulletin of the Korean Mathematical Society, vol. 49, no. 3, pp. 621-633, 2012. 


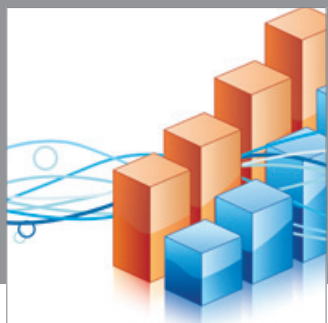

Advances in

Operations Research

mansans

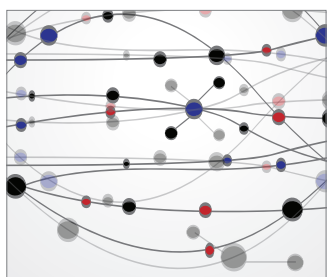

The Scientific World Journal
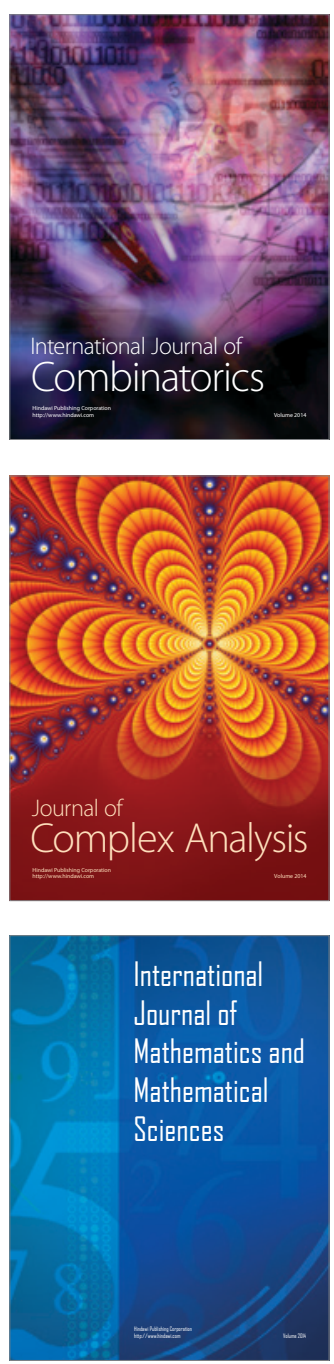
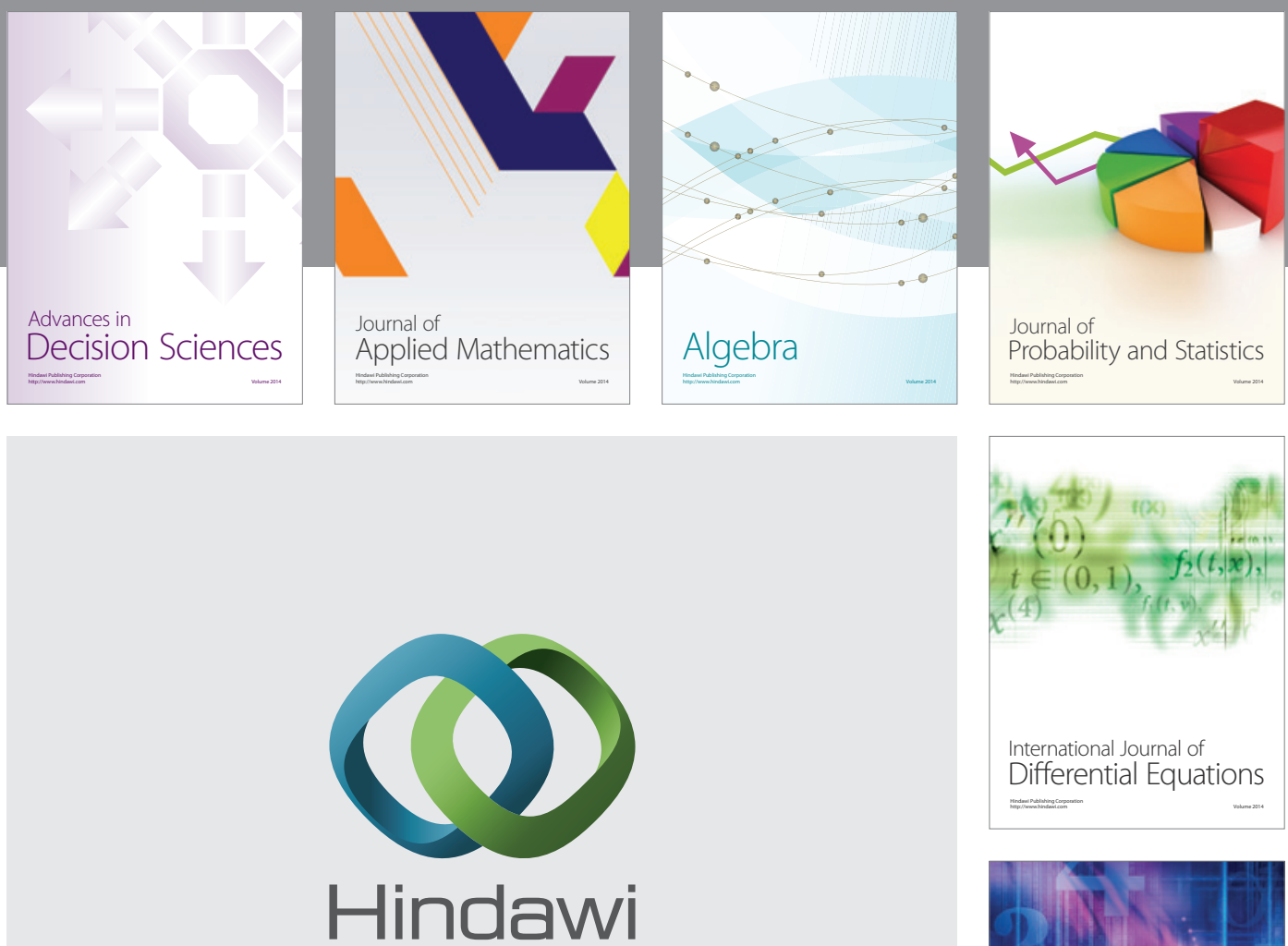

Submit your manuscripts at http://www.hindawi.com
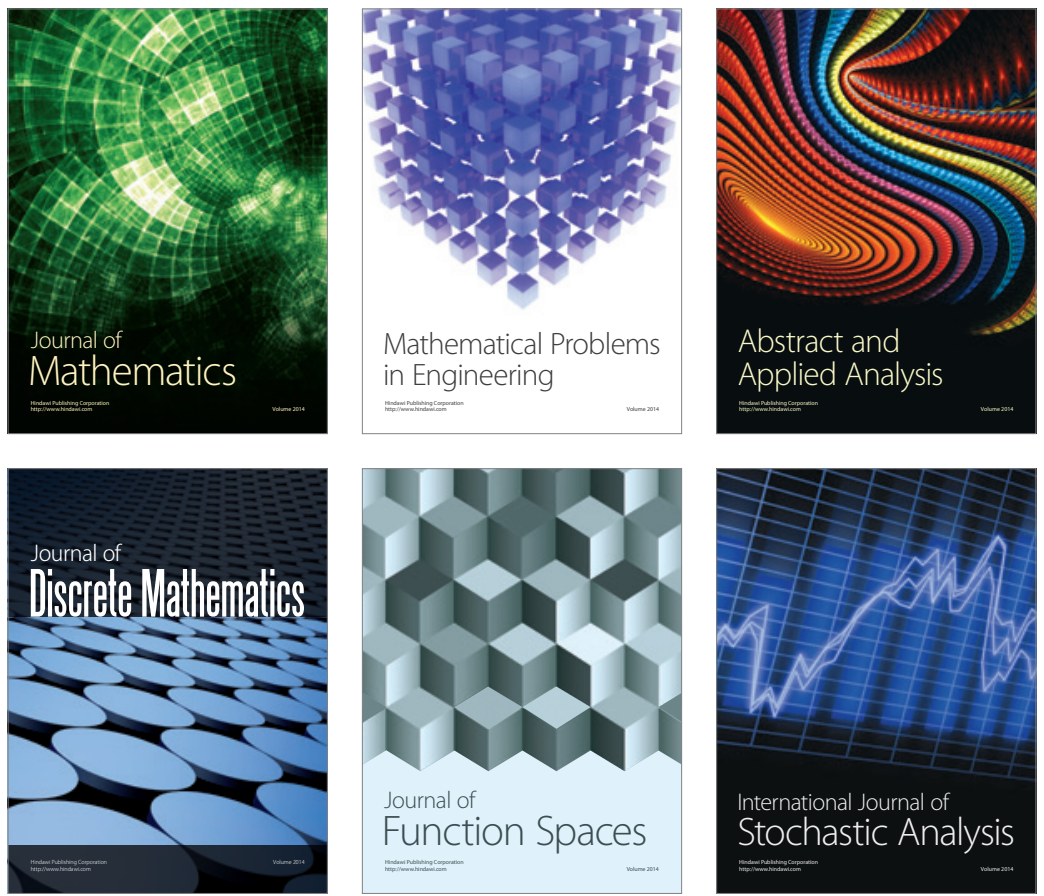

Journal of

Function Spaces

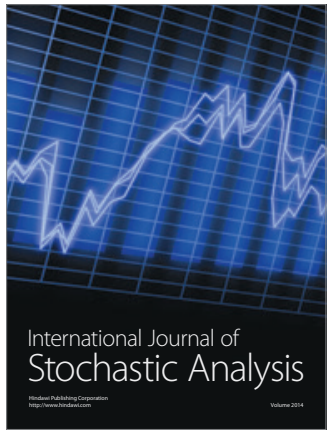

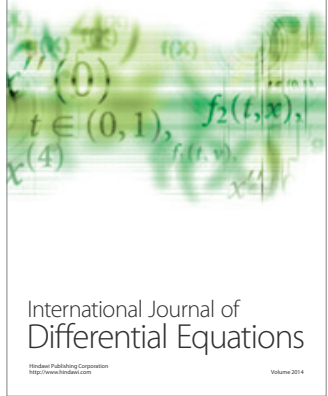
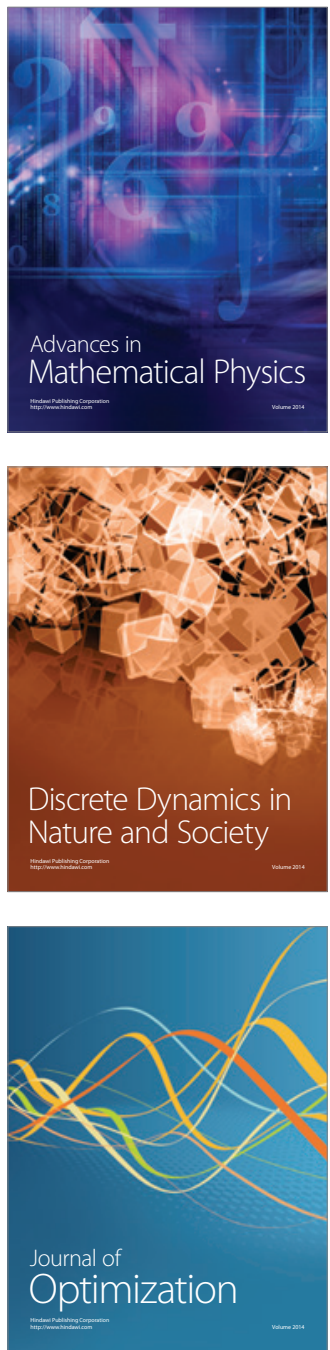